\section{In vitro antibiotic susceptibility patterns of bacterial keratitis isolates in Oxford, UK: a 10-year review}

\section{Abstract}

Purpose To analyse the spectrum of bacterial keratitis isolates and their in vitro antibiotic susceptibilities over a 10 -year period in Oxford, UK; and to compare the in vitro efficacy of ciprofloxacin with that of the combination of gentamicin and cefuroxime over the same period.

Methods All culture-positive corneal scrapes received from the Oxford Eye Hospital between July 1999 and June 2009 were identified retrospectively using a local microbiology database. For analysis of trends over time, the data was split into two equal 5-year periods. Statistical analysis was done using the $\chi^{2}$ and Fisher exact tests. Results Over the 10-year study period, 467 corneal scrapes were performed of which $252(54.0 \%)$ had positive bacterial cultures, growing a total of 267 organisms. The most commonly isolated bacteria were

Staphylococci $(\mathbf{4 0 . 1 \% )}$ ) followed by Pseudomonas species (28.5\%), other Gramnegative species $\mathbf{( 1 7 . 2 \% )}$, Streptococci $(\mathbf{7 . 1 \%})$, and Corynebacteria (6.0\%). Between the first and second time periods there was an increase in the number of coagulase-negative Staphylococci and an increased resistance of the non-Pseudomonas Gram-negative group to chloramphenicol. Of the $\mathbf{1 8 9}$ isolates tested for sensitivity to both empirical antibiotic regimens, $176(93.2 \%)$ were susceptible to ciprofloxacin whereas $188(99.5 \%)$ were susceptible to either gentamicin or cefuroxime $(P=0.0015)$.

Conclusions The spectrum of bacterial keratitis isolates and their in vitro antibiotic sensitivity patterns have generally remained stable over time. The combination of gentamicin and cefuroxime provides a broader
HO Orlans ${ }^{1}$, SJ Hornby ${ }^{1}$ and ICJW Bowler ${ }^{2}$

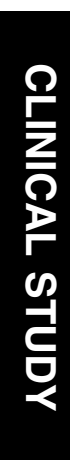

spectrum of antimicrobial cover than ciprofloxacin monotherapy in Oxford, although both regimens continue to be appropriate choices for the initial management of this condition.

Eye (2011) 25, 489-493; doi:10.1038/eye.2010.231; published online 21 January 2011

Keywords: keratitis; bacteria; antibiotic; resistance

'Oxford Eye Hospital, John Radcliffe Hospital, Oxford, UK

\section{Introduction}

Bacterial keratitis is a potentially devastating ocular infection. Topical antibiotics are used empirically before culture and sensitivity data become available. The antibiotic regimen chosen for the empirical phase of treatment must be of sufficiently broad spectrum to cover likely pathogens, and as bacterial prevalence and antibiotic sensitivities vary by place and over time, this choice should ideally be based upon timely and geographically-specific epidemiological data. Traditionally, a combination of fortified antibiotics - most commonly gentamicin with cefuroxime-has been used for this purpose. Fluoroquinolone monotherapy is now commonly used, with randomized controlled trials demonstrating non-inferiority and fewer side effects compared with combination therapy. ${ }^{1,2}$ Recently, a number of studies have demonstrated emerging resistance to many of these antibiotics, particularly to the fluoroquinolones, which may impact on their utility as empirical agents. ${ }^{3-5}$ In this study, we sought to review the microbiology of bacterial keratitis in Oxford over the past decade with a number of aims: first, to determine the bacteria associated with
${ }^{2}$ Department of Microbiology and Infectious Disease, John Radcliffe Hospital, Oxford, UK

Correspondence: HO Orlans, c/o SJ Hornby, Oxford Eye Hospital, West Wing, John Radcliffe Hospital, Headley Way, Headington, Oxford OX3 9DU, UK Tel: + 44 (0)7791 062913. E-mail: harry.orlans@ nhs.net

Received: 7 September 2010 Accepted in revised form: 2 December 2010 Published online: 21 January 2011

This work has previously been presented as a rapid fire presentation at the Oxford Ophthalmological Congress 2010 and as a poster at the Royal College of Ophthalmologists Annual Congress 2010 
keratitis and their antibiotic susceptibility profiles; second, to identify any trends over time; and third, to compare the in vitro efficacy of ciprofloxacin with that of the combination of gentamicin and cefuroxime.

\section{Materials and methods}

All culture-positive corneal scrapes received from the Oxford Eye Hospital between 1 July 1999 and 30 June 2009 were identified retrospectively using a local microbiology database. Corneal scrapes were taken with a 25-guage needle under proxymetacaine topical anaesthesia. Samples were immediately inoculated onto slides for Gram staining and microscopy, and onto blood, chocolate, and Sabouraud agar plates (in that order) for culture. Plates were incubated at $37^{\circ} \mathrm{C}$ in air with $5 \%$ carbon dioxide for a period of 5 days. Any microbial growth was regarded as significant and organisms were identified using standard laboratory techniques. In vitro antibiotic susceptibilities were determined by the modified Stokes' comparative disc method. ${ }^{6}$ The selection of antibiotics tested in this fashion was determined by the identity of the organism according to local protocols. The sensitivity testing process was controlled through both internal quality assurance and externally by the UK National External Quality Assurance Service (UK NEQAS) with satisfactory performance.

Where more than one corneal scrape had been taken from the same eye of a patient, subsequent isolates were only included in our analysis if the bacterial species and/or its antibiotic sensitivity profile differed from that identified in the initial sample. An isolate was considered to be resistant to combination therapy in vitro if resistance was demonstrated to both gentamicin and cefuroxime when these antibiotics were tested individually. For the purposes of analysis, the bacterial species were divided into five groups: Staphylococci, Pseudomonas species, Non-Pseudomonas Gram-negative species, Streptococci, and Corynebacteria. In order to identify any trends over time, the data was split into two equal 5-year periods with the first period defined between 1st July 1999 and 30th June 2004 and the second between 1st July 2004 and 30th June 2009.

The $\chi^{2}$ and Fisher exact tests were used for statistical analysis throughout. All calculations were performed using SPSS 16.0 statistical software (SPSS Inc., Chicago, IL, USA). A $P$-value of $<0.05$ was considered statistically significant.

\section{Results}

Over the 10-year study period, 467 corneal scrapes were performed. Of these, $252(54.0 \%)$ yielded positive bacterial cultures growing a total of 267 organisms. The range of bacteria isolated from corneal scrapes is shown it Table 1 . The most commonly isolated bacteria were Staphylococci $(40.1 \%)$ followed by Pseudomonas species $(28.5 \%)$, other Gram-negative species $(17.2 \%)$, Streptococci (7.1\%), and Corynebacteria (6.0\%). Together these five groups accounted for $98.9 \%$ of the isolates. Coagulasenegative Staphylococci, Pseudomonas aeruginosa, and Staphylococcus aureus were the most frequently encountered species, comprising 24.8, 24.3, and $12.4 \%$ of the cultures respectively. The total number of Gram-positive isolates was 145 (54.3\%).

There were no significant changes in the prevalence of bacteria from the five aforementioned groups between the first and second study periods. However, within the Staphylococcal group, an increase in the frequency of coagulase-negative Staphylococci was observed $(P=0.027)$.

Table 2 shows the in vitro susceptibility of Gram-positive and Gram-negative bacteria to a range of antibiotics in each study period. The rate of resistance to chloramphenicol amongst Gram-negative species increased from $46.3 \%$ in the first study period to $75.0 \%$ in the second $(P=0.002)$. The proportion of Gram-positive isolates resistant to ampicillin fell from 59.4 to $38.7 \%$ $(P=0.018)$. Other changes in the resistance profiles of Gram-positive and Gram-negative bacteria failed to reach statistical significance. The only significant change in the antibiotic resistance profiles of the five bacterial groups was an increase in the proportion of the non-Pseudomonas Gram-negative group resistant to chloramphenicol from 4.2 to $42.9 \%$ ( $P=0.003$ ).

Of the 189 isolates tested for sensitivity to both empirical antibiotic regimens, 176 (93.2\%) were susceptible to ciprofloxacin whereas 188 (99.5\%) were susceptible to the combination of gentamicin and cefuroxime $(P=0.0015)$. No increase in the resistance of bacteria to either regimen was observed between the two time periods of this study.

\section{Discussion}

The early empirical use of broad spectrum antibiotics in bacterial keratitis is essential to minimize damage to the cornea. Antibiotic choice should be guided by the local prevalence of pathogens, which varies by geographical location. In a recent study of microbial keratitis in Hyderabad, India, ${ }^{7} 42.3 \%$ of bacterial infections were caused by Staphylococcus epidermidis making this the most commonly isolated single species, as in our study. However, the incidence of keratitis caused by Pseudomonas species in our study was $30.3 \%$ compared with only $12.0 \%$ in Hyderabad. As contact lensassociated keratitis is commonly caused by Pseudomonas 
Table 1 Prevalence of bacteria isolated from corneal scrapes

\begin{tabular}{|c|c|c|c|c|}
\hline Bacteria & First period n (\%) & Second period n (\%) & All 10 years $\mathrm{n}(\%)$ & P-value \\
\hline Gram-positive cocci & $66(47.5)$ & $60(46.9)$ & $126(47.2)$ & 0.921 \\
\hline Coagulase-negative Staphylococci & $28(20.1)$ & $41(32.0)$ & $69(25.8)$ & $0.027^{*}$ \\
\hline MSSA & $22(15.8)$ & $11(8.6)$ & $33(12.4)$ & 0.073 \\
\hline MRSA & $4(2.9)$ & $1(0.8)$ & $5(1.9)$ & 0.372 \\
\hline Streptococcus pneumoniae & $5(3.6)$ & $3(2.3)$ & $8(3.0)$ & 0.724 \\
\hline$\alpha$-haemolytic Streptococci & $3(2.2)$ & $2(1.6)$ & $5(1.9)$ & 1.000 \\
\hline Enterococcus faecalis & $1(0.7)$ & $1(0.8)$ & $2(0.7)$ & 1.000 \\
\hline 'Viridans' Streptococci & $1(0.7)$ & $0(0)$ & $1(0.4)$ & 1.000 \\
\hline Streptococcus pyogenes & $1(0.7)$ & $0(0)$ & $1(0.4)$ & 1.000 \\
\hline Other Streptococcus spp. & $1(0.7)$ & $1(0.8)$ & $2(0.7)$ & 1.000 \\
\hline Gram-positive bacilli & $12(8.6)$ & $7(5.5)$ & $19(7.1)$ & 0.350 \\
\hline Corynebacterium spp. & $9(6.5)$ & $7(5.5)$ & $16(6.0)$ & 0.930 \\
\hline Propionibacterium spp. & $1(0.7)$ & $0(0)$ & $1(0.4)$ & 1.000 \\
\hline Bacillus spp. & $1(0.7)$ & $0(0)$ & $1(0.4)$ & 1.000 \\
\hline Other Gram-positive bacilli & $1(0.7)$ & $0(0)$ & $1(0.4)$ & 1.000 \\
\hline Gram-negative cocci & $5(3.6)$ & $5(3.9)$ & $10(3.7)$ & 1.000 \\
\hline Moraxella catarrhalis & $4(2.9)$ & $3(2.3)$ & $7(2.6)$ & 1.000 \\
\hline Other Moraxella spp. & $1(0.7)$ & $2(1.6)$ & $3(1.1)$ & 0.609 \\
\hline Gram-negative bacilli & $56(40.3)$ & $56(43.8)$ & $112(41.9)$ & 0.567 \\
\hline Pseudomonas aeruginosa & $29(20.9)$ & $36(28.1)$ & $65(24.3)$ & 0.167 \\
\hline Other Pseudomonas spp. & $7(5.0)$ & $4(3.1)$ & $11(4.1)$ & 0.634 \\
\hline Serratia marcescens & $2(1.4)$ & $0(0)$ & $2(0.7)$ & 0.499 \\
\hline Serratia liquefaciens & $1(0.7)$ & $0(0)$ & $1(0.4)$ & 1.000 \\
\hline Other Serratia spp. & $2(1.4)$ & $0(0)$ & $2(0.7)$ & 0.499 \\
\hline Haemophilus influenzae & $2(1.4)$ & $0(0)$ & $2(0.7)$ & 0.499 \\
\hline Escherichia coli & $1(0.7)$ & $1(0.8)$ & $2(0.7)$ & 1.000 \\
\hline Acinetobacter lwoffii & $2(1.4)$ & $0(0)$ & $2(0.7)$ & 0.499 \\
\hline Other Acinetobacter spp. & $1(0.7)$ & $0(0)$ & $1(0.4)$ & 1.000 \\
\hline Proteus mirabilis & $0(0)$ & $1(0.8)$ & $1(0.4)$ & 0.479 \\
\hline Pasteurella multocida & $1(0.7)$ & $0(0)$ & $1(0.4)$ & 1.000 \\
\hline Klebsiella spp. & $1(0.7)$ & $0(0)$ & $1(0.4)$ & 1.000 \\
\hline Aeromonas spp. & $1(0.7)$ & $0(0)$ & $1(0.4)$ & 1.000 \\
\hline Other Gram-negative bacilli & $6(4.3)$ & $14(10.9)$ & $20(7.5)$ & 0.061 \\
\hline Total & 139 & 128 & 267 & \\
\hline
\end{tabular}

Abbreviations: MSSA, Methicillin-sensitive Staphylococcus aureus; MRSA, Methicillin-resistant Staphylococcus aureus.

The final column ( $P$-value) shows the statistical significance of two-tailed $\chi^{2}$ or Fisher exact tests comparing data from the First and Second study periods. ${ }^{*} P<0.05$.

Table 2 In vitro susceptibility of Gram-positive and Gram-negative bacteria to antibiotics in the two study periods and over the entire 10 years

\begin{tabular}{|c|c|c|c|c|c|c|}
\hline \multirow[t]{2}{*}{ Antibiotic } & \multicolumn{3}{|c|}{ Gram-positive } & \multicolumn{3}{|c|}{ Gram-negative } \\
\hline & First period & Second period & All 10 years & First period & Second period & All 10 years \\
\hline Ciprofloxacin & $38 / 46(82.6 \%)$ & $38 / 43(88.4 \%)$ & $76 / 89(85.4 \%)$ & $50 / 51(98.0 \%)$ & $51 / 51(100 \%)$ & $101 / 102(99.0 \%)$ \\
\hline Gentamicin & $63 / 74(85.1 \%)$ & 60/67 (89.6\%) & 123/141 (87.2\%) & $59 / 59(100 \%)$ & $60 / 60(100 \%)$ & 119/119 (100\%) \\
\hline Cefuroxime & $56 / 68(82.4 \%)$ & $50 / 63(79.4 \%)$ & 106/131 (80.9\%) & 7/51 (13.7\%) & $2 / 52(3.8 \%)$ & 9/103 (8.7\%) \\
\hline Chloramphenicol & $64 / 71(90.1 \%)$ & $63 / 66(95.5 \%)$ & $127 / 137(92.7 \%)$ & $29 / 54(53.7 \%)$ & $15 / 60(25.0 \%)^{*}$ & $44 / 114(38.6 \%)$ \\
\hline Ampicillin & $28 / 69(40.6 \%)$ & $38 / 62(61.3 \%)^{*}$ & $66 / 131(50.4 \%)$ & $\mathrm{N} / \mathrm{A}$ & $\mathrm{N} / \mathrm{A}$ & $\mathrm{N} / \mathrm{A}$ \\
\hline Vancomycin & $49 / 49(100 \%)$ & $45 / 45(100 \%)$ & 94/94 (100\%) & $\mathrm{N} / \mathrm{A}$ & $\mathrm{N} / \mathrm{A}$ & $\mathrm{N} / \mathrm{A}$ \\
\hline Polymyxin B & $\mathrm{N} / \mathrm{A}$ & $\mathrm{N} / \mathrm{A}$ & $\mathrm{N} / \mathrm{A}$ & $47 / 55(85.5 \%)$ & $53 / 60(88.3 \%)$ & $100 / 115(87.0 \%)$ \\
\hline Ceftazidime & $\mathrm{N} / \mathrm{A}$ & $\mathrm{N} / \mathrm{A}$ & $\mathrm{N} / \mathrm{A}$ & $38 / 38(100 \%)$ & $40 / 40(100 \%)$ & $78 / 78(100 \%)$ \\
\hline
\end{tabular}

In each cell, the number of susceptible bacteria is shown alongside the total number tested against that antibiotic in that time period. These proportions are also expressed as percentages. Only selected antibiotics were tested against specific isolates in accordance with local protocols. ${ }^{*} P<0.05$ versus corresponding 'First period' by the $\chi^{2}$-test. 
aeruginosa, ${ }^{8}$ this difference may be attributable to the higher frequency of contact lens wear as a risk factor within the UK. Another study conducted at a university hospital in Taiwan ${ }^{9}$ revealed a very different spectrum of bacterial isolates. Here coagulase-negative Staphylococci were infrequently encountered (6.2\%), and Pseudomonas aeruginosa represented a much greater proportion of the data set $(46.7 \%)$. By contrast, in an investigation conducted within six specialist ophthalmology centres in the UK between 2003 and 2006, the spectrum of microbial cultures from bacterial keratitis was remarkably similar to that which we report here with coagulase-negative Staphylococci, Pseudomonas species, and Staphylococcus aureus comprising 27,21 , and $13 \%$ of the isolates respectively. ${ }^{10}$

In our study, $54.0 \%$ of corneal scrapes yielded positive cultures in the laboratory. One potential explanation for this relatively low culture rate may be the frequent use of topical antimicrobial therapy within primary care before emergency ophthalmic referral and corneal scraping. This figure is however comparable with that attained in other centres. $5,7,9$

Fluoroquinolones are currently popular choices for empiric therapy in bacterial keratitis owing to their broad spectrum of activity, low toxicity, and high level of absorption at the ocular surface. However, emerging resistance to this class of antibiotics has been increasingly noted in a number of centres over the last 20 years. Recently, Afshari et al reported an increase in the resistance of Gram-positive keratitis isolates to ciprofloxacin from 12 to $22 \%$ over 10 month periods in consecutive years. ${ }^{3}$ Goldstein et al found large increases in the rates of in vitro resistance to both ciprofloxacin and ofloxacin among Staphylococcus aureus isolates between 1993 and 1997. ${ }^{4}$ The authors also found high rates of resistance to these antibiotics in Streptococcus and coagulase-negative Staphylococcus isolates. Similarly, Alexandrakis and colleagues reported an increase in the resistance of Staphylococcus aureus isolates to ciprofloxacin from $11 \%$ in 1990 to $28 \%$ in $1998 .{ }^{5}$ Our data demonstrate no such trend. As Table 2 shows, the rate of resistance to ciprofloxacin among Gram-positive species was $17.4 \%$ in the first study period and $11.6 \%$ in the second $(P=0.55)$, whereas only one Gram-negative isolate was resistant to ciprofloxacin over the entire 10-year period.

Aminoglycosides such as gentamicin are commonly used as alternatives to fluoroquinolones in the empiric treatment of bacterial keratitis, often in combination with a cephalosporin to increase Gram-positive activity, particularly against Streptococci. In our study the rate of resistance to gentamicin did not change significantly over time, a finding consistent with reports from elsewhere. $^{5}$
The proportion of Gram-negative organisms resistant to chloramphenicol increased from $46.3 \%$ in the first half of our study to $75.0 \%$ in the second $(P=0.002)$. This trend was most evident in the non-Pseudomonas Gramnegative sub-group $(P=0.003)$. Chloramphenicol was however active against Gram-positive organisms with $92.7 \%$ of isolates demonstrating susceptibility in vitro over the 10-year period. Tuft and Matheson described similar changes in chloramphenicol resistance patterns in bacterial keratitis isolates between 1985 and 1999 in London. ${ }^{11}$ However, our study based in a secondary care setting may have exaggerated the true proportion of keratitis isolates resistant to chloramphenicol. Topical preparations of chloramphenicol are commonly prescribed by primary care physicians in the UK, and such preparations are now freely available on an over-the-counter basis at pharmacies. Topical use of chloramphenicol before sampling of the cornea at specialist ophthalmic centres may select for bacteria resistant to this antibiotic, making these organisms more likely to culture in the laboratory.

Although both of the empirical regimens studied demonstrated in vitro activity against a broad spectrum of corneal organisms, the combination of gentamicin and cefuroxime was significantly more likely to be active than ciprofloxacin alone $(P=0.0015)$. Given the more favourable side effect profile and convenience, however, treatment with a fluoroquinolone remains a reasonable choice for first-line management of bacterial keratitis.

The conclusions of this study must be considered in the context of its methodological limitations. It is possible that in vitro resistance data, as used for this investigation, may underestimate the true efficacy of treatment in clinical practice, as the concentration of drug achieved at the ocular surface is potentially much greater than that used for in vitro susceptibility testing. The results of in vitro antimicrobial testing have however been shown to be predictive of therapeutic response in fluoroquinolone-treated bacterial keratitis. ${ }^{12}$ Laboratory results should nevertheless be used only to guide continuing therapy in cases of poor clinical response to empirical management. It must be noted that a large proportion of causative organisms could not be included in this study by virtue of their failure to culture in the microbiology laboratory. Further, we cannot exclude the possibility that bacteria identified from a proportion of culture-positive corneal scrapes, for example a subset of the coagulase-negative Staphylococci, may represent non-pathogenic commensal species from the ocular surface. Over the time period of this study, cultures from corneal scrapes were tested against ciprofloxacin as a representative antibiotic of the fluoroquinolone class. Susceptibility data for the newer fluoroquinolones ofloxacin, gatifloxacin, and moxifloxacin, which are 
increasingly used in this setting, were not available for this retrospective study. The antibacterial activity and rates of resistance to the various fluoroquinolones in keratitis isolates are not identical. Although ciprofloxacin has lower mean inhibitory concentrations than ofloxacin for organisms commonly associated with keratitis, it does not penetrate the cornea as readily. ${ }^{13}$ A recent study from China reported a greater level of in vitro resistance of bacterial keratitis isolates to ciprofloxacin than to ofloxacin. ${ }^{14}$ Kowalski et al demonstrated that fourth generation fluoroquinolones were effective in vitro against Staphylococcus aureus isolates resistant to ciprofloxacin, ofloxacin, and levofloxacin. ${ }^{15}$ Finally, as the range of pathogenic bacteria varies greatly in different regions across the world, our data cannot be extrapolated universally.

Notwithstanding these limitations, a number of conclusions may be drawn from this study. First, the spectrum of bacteria associated with culture-positive infective keratitis has not, in general, changed significantly between 1999 and 2009 in Oxford. Second, although this spectrum is comparable to that reported from centres within the UK, it differs markedly from data published from other regions of the world. Third, resistance to chloramphenicol is increasing amongst Gram-negative bacteria. Finally, the combination of gentamicin and cefuroxime has a broader spectrum of activity than ciprofloxacin monotherapy against culturepositive isolates in vitro. However, both regimens were active against most organisms isolated, and are still appropriate choices for empirical therapy.

\section{Conflict of interest}

The authors declare no conflict of interest.

\section{Acknowledgements}

The authors would like to acknowledge Jane Simms for her assistance with initial data extraction.

\section{References}

1 O'Brien TP, Maguire MG, Fink NE, Alfonso E, McDonnell P, The Bacterial Keratitis Study Research Group. Efficacy of ofloxacin $v$ s cefazolin and tobramycin in the therapy for bacterial keratitis. Arch Ophthalmol 1995; 113: 1257-1265.

2 Khokhar S, Sindhu N, Mirdha BR. Comparison of topical $0.3 \%$ ofloxacin to fortified tobramycin-cefazolin in the therapy of bacterial keratitis. Infection 2000; 28(3): 149-152.

3 Afshari NA, Ma JJK, Duncan SM, Pineda R, Starr CE, Decroos FC et al. Trends in resistance to ciprofloxacin, cefazolin, and gentamicin in the treatment of bacterial keratitis. J Ocul Pharmacol Ther 2008; 24: 217-223.

4 Goldstein MH, Kowalski RP, Gordon YJ. Emerging fluoroquinolone resistance in bacterial keratitis: a 5 year review. Ophthalmology 1999; 106: 1313-1318.

5 Alexandrakis G, Alfonso EC, Miller D. Shifting trends in bacterial keratitis in South Florida and emerging resistance to fluoroquinolones. Ophthalmology 2000; 107: 1497-1502.

6 Brown DFJ, Kothari D. Antimicrobial-susceptibility testing of rapidly growing pathogenic bacteria. II. A field trial of four disc-diffusion methods. J Antimicrob Chemother 1978; 4: 27-38.

7 Gopinathan U, Sharma S, Garg P, Rao GN. Review of epidemiological features, microbiological diagnosis and treatment outcome of microbial keratitis: experience of over a decade. Indian J Ophthalmol 2009; 57: 273-279.

8 Alfonso E, Mandelbaum S, Fox MJ, Forster RK. Ulcerative keratitis associated with contact lens wear. Am J Ophthalmol 1986; 101(4): 429-433.

9 Fong CF, Hu FR, Tseng CH, Wang IJ, Chen WL, Hou YC. Antibiotic susceptibility of bacterial isolates from bacterial keratitis cases in a university hospital in Taiwan. Am J Ophthalmol 2007; 144: 682-689.

10 Kaye S, Tuft S, Neal T, Tole D, Leeming J, Figueiredo F et al. Bacterial susceptibility to topical antimicrobials and clinical outcome in bacterial keratitis. Invest Ophthalmol Vis Sci 2010; 51: 362-368.

11 Tuft SJ, Matheson M. In vitro antibiotic resistance in bacterial keratitis in London. Br J Ophthalmol 2000; 84: 687-691.

12 Wilhelmus KR, Abshire RL, Schlech BA. Influence of fluoroquinolone susceptibility on the therapeutic response of fluoroquinolone-treated bacterial keratitis. Arch Ophthalmol 2003; 121(9): 1229-1233.

13 Diamond JP, White L, Leeming JP, Hoh HB, Easty DL. Topical $0.3 \%$ ciprofloxacin, norfloxacin, and ofloxacin in treatment of bacterial keratitis: a new method for comparative evaluation of ocular drug penetration. Br J Ophthalmol 1995; 79: 606-609.

14 Zhang C, Liang Y, Deng S, Wang Z, Li R, Sun X. Distribution of bacterial keratitis and emerging resistance to antibiotics in China from 2001 to 2004. Clin Ophthalmol 2008; 2: 275-579.

15 Kowalski RP, Dhaliwal DK, Karenchak LM, Romanowski EG, Mah FS, Ritterband DC et al. Gatifloxacin and moxifloxacin: an in vitro susceptibility comparison to levofloxacin, ciprofloxacin, and ofloxacin using bacterial keratitis isolates. Am J Ophthalmol 2003; 136: 500-505. 